\title{
La nature urbaine: dégradation quantitative et qualitative des espaces verts urbains, cas de la ville steppique de M'Sila, Algérie
}

Natureza urbana: degradação quantitativa e qualitativa de espaços verdes

urbanos, caso da cidade de estepe de M'sila, Argélia

Urban nature: quantitative and qualitative degradation of urban green spaces, case of the steppe city of M'sila, Algeria

Mohamed Mili[a] (1), Hynda Boutabba[a], Samir-Djemoui Boutabba[a]

[a] University of M'sila, M'sila, Algeria

Comment citer: Mili, M., Boutabba, H., \& Boutabba, S-D. (2019). La nature urbaine: dégradation quantitative et qualitative des espaces verts urbains, cas de la Ville Steppique de M'sila, Algérie. urbe. Revista Brasileira de Gestão Urbana, 11, e20180138. https://doi.org/10.1590/2175-3369.011.e20180138

\section{Resume}

Le rôle de la nature dans une ville qui commence à prendre de l'ampleur en matière d'extension urbaine au dépens de ces espaces verts reste un sujet très peu développé dans les multiples études urbaines réalisées sur la ville de M'sila. En effet le centre de cette agglomération, dense de ses multiples lotissements et cités collectives d'habitat social, est toujours présenté comme un milieu hostile à la nature. Pourtant cette ville a été pendant longtemps un des greniers de blé et d'abricotiers de toute la région steppique du Hodna.En utilisant une approche historique et la technique de l'entretien "non-directif" qui favorise un déplacement du questionnement tourné vers le savoir et les expériences propres des acteurs publics sociaux ce papier tente de montrer la situation actuelle de la nature dans une ville algérienne caractérisée par un climat semi-aride ainsi que la manière de sa gestion par les différents acteurs de la ville.

Mots-clés: Espace-vert. Gestion. Ratio espace-vert par habitant. Parcours historique. Dégradation. Ville de M'sila. 


\section{Resumo}

O papel da natureza numa cidade que está começando a crescer em termos de extensão urbana em detrimento dos espaços verdes continua sendo um assunto pouco desenvolvido nos múltiplos estudos urbanísticos realizados na cidade de M'sila. Com efeito, o centro dessa aglomeração, denso pelas diferentes subdivisões e conjuntos habitacionais coletivos, é sempre apresentado como um ambiente hostil à natureza. No entanto, essa cidade tem sido por muito tempo um dos celeiros de trigo e pomares de damascos da região de estepe de Hodna. Utilizando uma abordagem histórica e a técnica de entrevista "não-diretiva", que favorece um questionamento voltado para o conhecimento e as experiências dos atores sociais, este trabalho procura mostrar a atual situação da natureza numa cidade argelina caracterizada por um clima semiárido, bem como a sua gestão pelos diferentes atores sociais da cidade.

Palavras-chave: Espaço verde. Gestão. Proporção espaço verde por habitante. Percurso histórico. Degradação. Cidade de M'sila.

\section{Abstract}

The role of nature in a city growing in terms of urban extension at the expense of green spaces, like M'sila, remains an underdeveloped subject in the many urban studies carried out on this city. Indeed, the center of this agglomeration, dense of its multiple subdivisions and collective social-housing estates, is always presented as a hostile environment to nature. However, this city was for a long time one of the granaries of wheat and apricot-tree of all the steppe region of Hodna. This paper aims at describing the current situation of nature in an Algerian city characterized by a semi-arid climate, as well as its management by the different social actors of the city. There were used a historical approach and a nondirective interview. This interview technique favors a questioning focused on the knowledge and experiences of the public and social actors.

Keywords: Green space. Management. Green space ratio by inhabitant. Historical course. Degradation. City of M'sila.

\section{Introduction}

L'infinité des séquences et des sensations que présente la nature urbaine peuvent nous offrir une satisfaction physique et visuelle, à contrario un malaise physique et visuel affecte négativement notre cadre de vie (Goodman, 1968; Laurie, 1986; Muret et al., 1987). Les préoccupations et la mise en place des politiques en matière d'espace vert s'opèrent à des volumes et rythmes différents d'un pays à l'autre. La norme conventionnelle internationale de $10 \mathrm{~m}^{2}$ d'espace vert par habitant (Planel, 1980) est loin d'être studieuse comparativement aux habitants de Paris qui bénéficient d'une moyenne de $14 \mathrm{~m}^{2} / \mathrm{h}$ et ceux de Berlin qui bénéficient de $21 \mathrm{~m}^{2} / \mathrm{h}$ (France, 2014).

En Algérie l'acteur de la ville dans son combat contre la crise du logement n'accordait d'importance qu'à la résorption de cette crise (Dahmani \& Moudjari, 2013; Hdjeidj et al., 2003; Hafiane, 1992) et ne se préoccupait pas de la qualité de l'espace non bâti végétaliséqui était resté pour très longtemps reléguée au dernier rang des priorités des gestionnaires de la ville (Ali-Khodja, 2011).

L'espace vert public de la ville de M'sila ne semble pas faire l'exception à la règle générale des espaces verts des autres villes d'Algérie (Ali-Khodja, 2000; Lamri, 2012) notamment ceux des régions steppiques. Le cadre vert de la ville de M'sila présente une logique d'organisation peu apparente et un cadre défavorable à l'épanouissement de l'individu accusant un aspect de sécheresse accentuant aussi une aridité du climat et une pollution visuelle. En réalité ces espaces verts avec leurs différentes catégories urbaines accusent non seulement la qualité mais aussi la quantité (Bekouche, 1999).

Dans la présente étude nous allons procéder à l'évaluation de l'état des espaces verts de la ville en terme quantitatif et qualitatif. L'objectif principal étant de détecter le rôle des organismes étatiques concernés par la gestion urbaine dans la dégradation de l'élément vert de cette ville. Pour cela nous 
allons procéder en trois temps. Dans une première phase, nous allons exposer l'évolution des espaces verts à travers un parcours historique sur l'urbanisation de la ville de M'sila. Dans une seconde tape, nous allons diagnostiquer la situation actuelle des différentes catégories du cadre vert dans cette localité. La troisième étape sera consacrée à l'indentification des différents organismes de gestion des espaces végétalisés dans cette ville ainsi que l'impact de leur gestion sur l'état des espaces verts et l'environnement urbain.

\section{Méthodologie}

Cette étude tente de dévoiler l'état déplorable des espaces verts urbains dans les villes steppiques algériennes à travers la ville de M'sila, capitale de la région du Hodna. Un de ses objectifs est de tirer la sonnette d'alarme et de sensibiliser l'opinion publique et les pouvoirs publics sur l'urgence de l'établissement d'un plan stratégique de préservation de la nature dans les zones urbaines. Pour cela nous avons choisi plusieurs approches et un ensemble de techniques de recherche selon les étapes analytiques abordées:

- Dans le premier volet analytique nous avons utilisé l'approche historique en faisant appel à un fond documentaire: les documents officiels coloniaux, notamment les rapports des généraux qui ont gouvernés le Hodna, les travaux de géographes (Despois, 1953; Sebhi, 1987), et les documents officiels de l'Algérie indépendante (PDAU révisé en Sétif, 2010; Loi 07-06 du 31.05.2007. relative aux espaces verts, Algérie, 2007).

- Le deuxième volet s'est appuyé sur une approche statistique où la superficie et le nombre des espaces verts de la ville ont été analysés et comparés au nombre d'habitant

- Le troisième volet a utilisé la technique de l'entretien non directif. le corpus nécessaire à la réalisation de l'enquête par entretien est d'une manière générale de taille réduite, dans la mesure où les informations issues des entretiens sont validées par le contexte et n'ont pas besoin de l'être par leur probabilité d'occurrence. Par ailleurs, ce corpus doit être, selon Becker (2002), diversifié reposant sur la sélection de composantes, non strictement représentatives, mais caractéristiques de la population. Ce qui est appelé par Donégani et al. (1980) de «principe de diversification». Pour cela nous avons choisi deux représentants par institution (mairie, direction de la conservation des forets, direction de l'environnement, les entreprises de réalisation des espaces verts et les représentants des associations de quartier) ce qui fait élever notre échantillon à 24 . Nous avons commencé par expliquer que nous nous intéressons aux espaces verts de la ville de M'sila. La consigne principale est: «en quoi consiste le rôle de votre institution dans la gestion et préservation des espaces verts de la ville?».

\section{Présentation de la zone d'étude}

La ville de M'sila constitue la capitale de la région du Hodna. Le territoire du Hodna s'étale sur une superficie de $6951 \mathrm{Km} 2$, au cœur de l'un des plus vastes ensembles de zones semi arides et steppiques qui existent en Afrique du Nord (Despois, 1953; Sebhi, 1987; Boutabba, 2013). Cette région est en retrait des cotes méridionales de la méditerranée entre l'Atlas Tellien au Nord et l'Atlas saharien au Sud (Voir Figure 1). Historiquement, M'sila était une ville à vocation pastorale et agricole, les jardins privés et vergers avaient longtemps forgé son identité. 


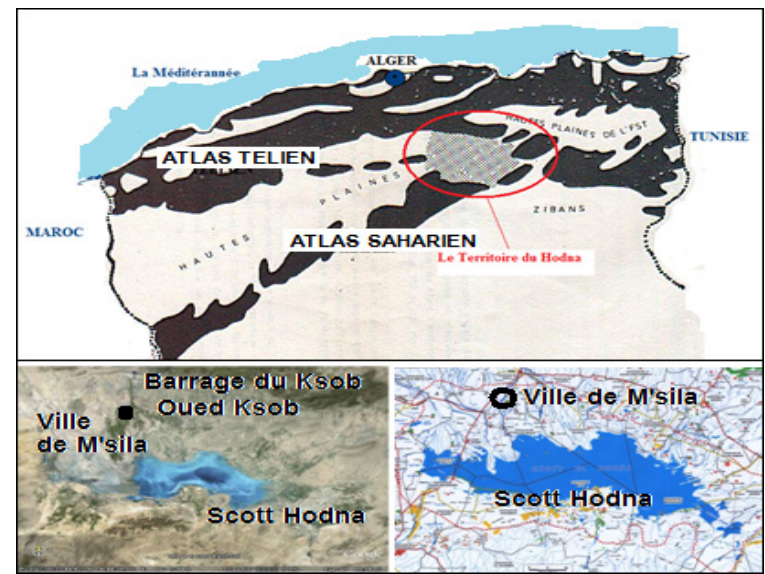

Figure 1 - Situation géographique de la région steppique du Hodna. Source: Sebhi, 1987; Conservation des forêts de M'sila, 2015.

\section{Evolution du "cadre vert" de la ville de M'sila à travers le temps}

A la période médiévale, M'sila centre d'origine antique (Despois, 1953) était un pays de cultures irriguées et inondées. Ibn Hawkal (1842) et Al Bakri (1913) parlaient "Des nombreux troupeaux du Hodna, de ses moutons, de ses chameaux, de ses bêtes de selles mais aussi des bovins qui pouvaient vivre de la steppe seule." Al Idrisi (1866) et Al Muqaddasi (1950) parlaient des véritables oasis qui bordaient cette ville et soulignaient le nombre et la variété des arbres fruitiers. A la veille du colonialisme, M'sila était formée de quatre groupements urbains situés à la rive Est du oued "Ksob": Um Ksar ${ }^{1}$ d'aspect saharien connu sous le nom de Kherbet Tellis (Despois, 1953) auquel s'est accolé plus tard trois autres quartiers les Dacherra de Chettaoua, Kraghla et Rass El Hara (Boutabba et al., 2014). Des agglomérations aux maisons accolées entourées de cultures denses de céréales et de jardins au milieu d'une steppe de pâturage maigre et de cultures extensives. Les habitants étaient dans leur majorité propriétaires terriens. Les céréales constituaient la richesse économique principale.

Pendant la colonisation française, aux alentour des années 1920-1930, l'administration coloniale avait choisi la rive opposée de l'Oued Ksob comme lieu d'implantation de son nouveau centre appelé par les autochtones "Edhahra" et parfois "Damier colonial". Cette petite implantation urbaine était érigée selon les mêmes techniques et outils urbains habituellement employés par la France dans ses colonies Nord africaines (Malverti \& Pinon, 1997; Almi, 2002) à savoir: L'usage des galeries le long des rues principales, la régularité, la proportion, la symétrie et l'ordre ainsi que les plantations d'arbres d'alignement considérées comme outil indispensable pour la hiérarchisation des réseaux des voies. Les deux voies principales d'Edhahra menant à Bordj Bou Arréridj-Boussaâda (l'actuelle RN 45) et à Taghmount (l'actuelle RN 60) étaient les premières d'avoir bénéficié d'arbres d'alignement de l'espèce Eucalyptus et faux poivrier (Boutabba et al., 2014), (Voir Figure 2).

\footnotetext{
${ }^{1}$ Selon le dictionnaire Larousse, le Ksar est un village fortifié de l'Afrique du Nord présaharienne, le long des oueds, au débouché des
} torrents montagnards. 

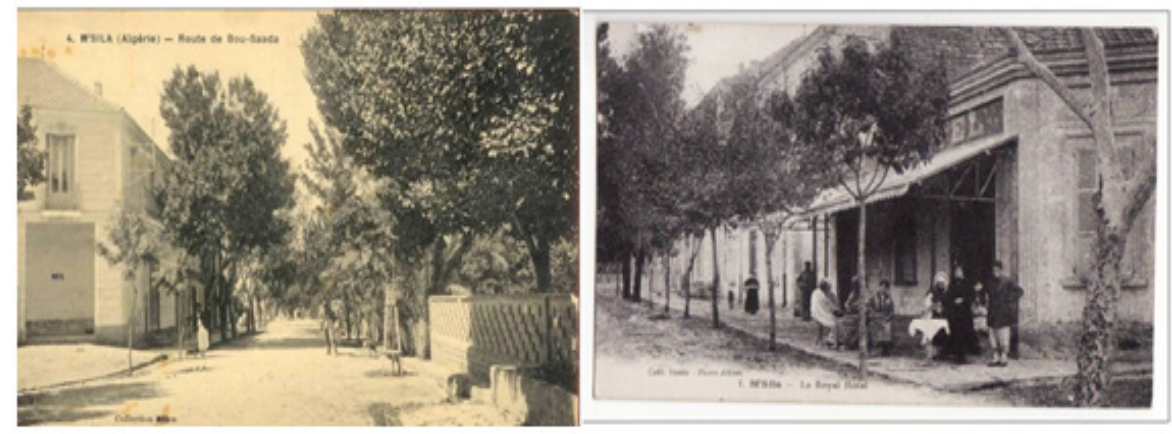

Figure 2 - Les arbres d'alignement des artères principales de la ville de M'sila pendant la période coloniale (1950). A gauche: Les allées route de Boussaâda. A droite: Les allées du Royal Hôtel. Source: Collection de l'étoile collection Albert (2017).

Les deux cités, arabe: la médina et coloniale: Edhahra ainsi matérialisées étaient jointées par des places publiques: la place Aristide Laussel du coté Sud limitrophe au quartier d'El Argoub et celle de la commune mixte du coté Nord limitrophe du quartier d'El Kouche (Voir Figure 3).

Ces dernières constituait probablement les premières places publiques proprement dites de la ville de M'sila à cette époque en plus de la place Bachagua Boudial, qui se situait au centre du quartier Edhahra. Ces places étaient des espaces d'embellissement, d'articulation ou de desserrement urbain, mais aussi des espaces où s'exprime, dans un contexte colonial, la sociabilité européenne (Kettaf, 2015).
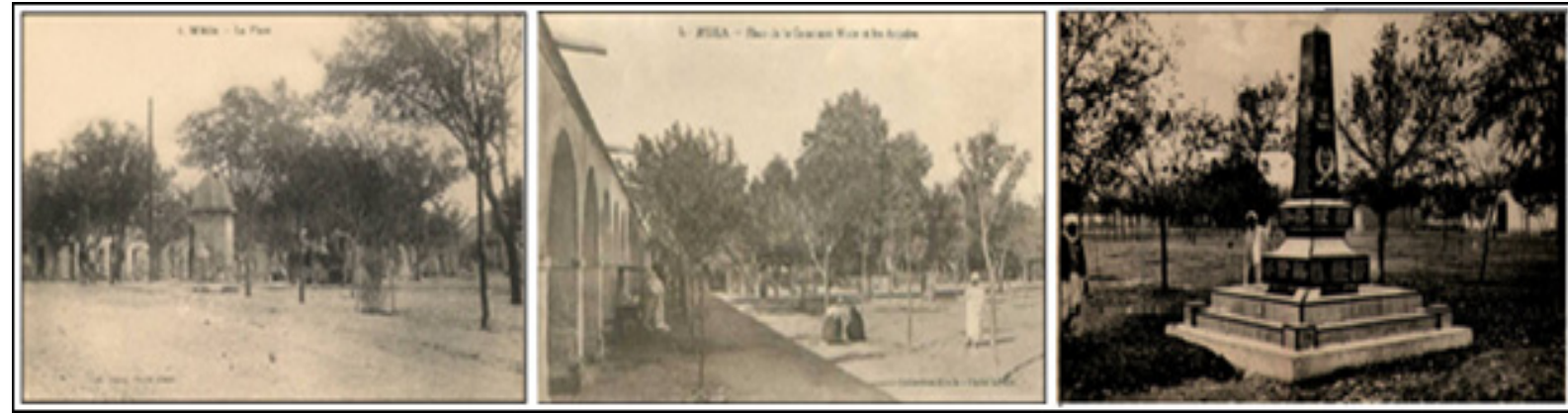

Figure 3 - Les places publiques de Msila à l'époque coloniale (1959). A gauche: Place aristide Laussel. Au milieu: Place de la commune mixte. A droite: Bachagua Boudial. Source: Collection de l'étoile collection Albert.

En 1946 et selon Rosso (2018), les 2367 hectares du centre de colonisation étaient ensemencés en céréales, les cultures maraîchères occupaient une quinzaine d'hectares, l'arboriculture connaissait un certain essor, 550 abricotiers, 260 pêchers sont en plein rendement avec d'autres espèces: figuiers, amandiers et pruniers. A l'aube de l'indépendance, la ville de M'sila a vu la destruction de son cadre bâti suite au séisme de 1965. A cette époque les autorités étaient occupées à reloger les habitants de la ville qui étaient à plus de $80 \%$ sans abri. Ceci était matérialisé par l'apparition de deux quartiers 300 logements et 500 logements ainsi que le lotissement "Ouaoua Madani" (Voir Figure 4). Cette extension urbaine s'était opérée au détriment des jardins d'abricots (Khoudour, 1999). 


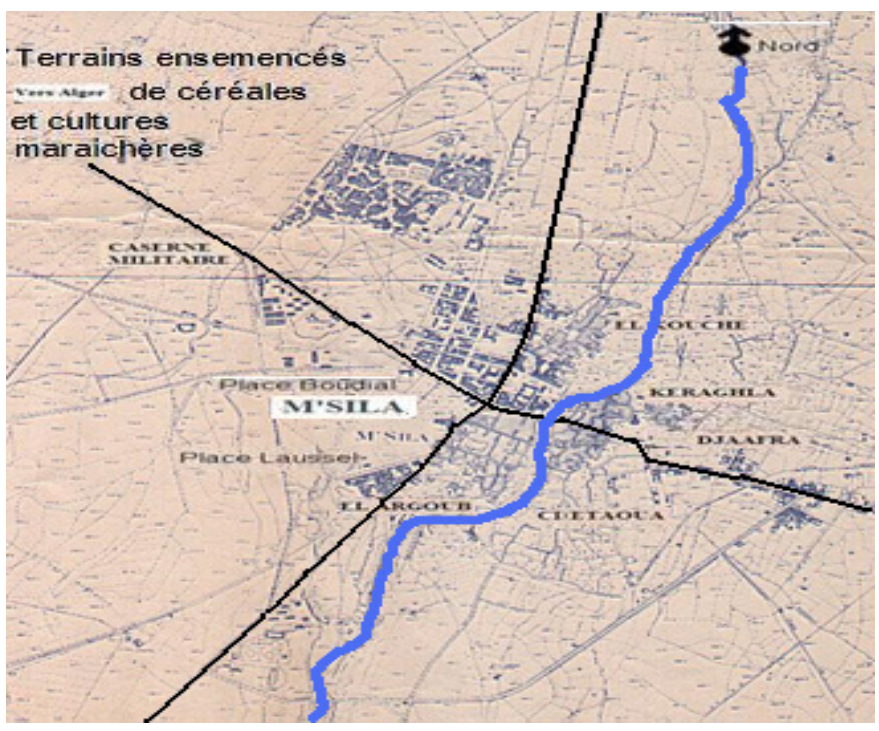

Figure 4 - Les quartiers et places publiques de la ville de M'sila pendant la période coloniale (1950). Source: Plan de la ville de M'sila, 1958.

Face à cette urgence à reloger les sinistrés, le cadre vert, était jugé comme un luxe et était relégué au dernier rang des priorités. En effet, pendant cette période aucun espace végétalisé n'a été créé. Les espaces verts de la ville coloniale étaient réaménagés et renommées. La place Boudial rebaptisée Ahmed Madeghri était dénuée de son monument aux morts, commémorant les soldats français de la première guerre mondiale pour être remplacée par un monument honorant les combattants de la guerre de libération nationale. La place Laussel était rebaptisé place des martyres Chouhada, (Voir Tableau 1).

En 1975 la ville de M'sila était promue au rang de wilaya. Elle s'était caractérisée par la naissance de grands projets urbains: deux zones urbaines nouvelles ZHUN 01 et 02 (Mili et al., 2016). Cette urbanisation s'est faite, une fois de plus, au détriment des terres agricoles et des jardins d'abricots, autrefois symboles de la ville. De 1975 jusqu'à 1979, la ville de M'sila a vu l'édification de prés de huit cités collectives (Mili, 2018) et d'un seul espace vert: le parc de la route de Boussaâda.

Tableau 1 - Espaces verts à l'aube de l'indépendance

\begin{tabular}{|c|c|c|c|}
\hline $\mathbf{N}^{\circ}$ & Ancienne désignation & Nouvelle nomination & Surface $\mathrm{m}^{2}$ \\
\hline 01 & place Aristide Laussel & Place des martyres «chouhada» & 1400.00 \\
\hline 02 & Place Bachaga Boudial & Place Ahmed Madeghri & 1600.00 \\
\hline 03 & Place de la Poste & Place Amirouchel & 700.00 \\
\hline 04 & Placette régie des impôts & Place Amirouche2 & 700.00 \\
\hline 05 & Place de la commune mixte & Place de l'APC & 1500.00 \\
\hline
\end{tabular}

Source: Mili \& Boutabba (2013).

De 1979 jusqu'à 1989, la ville de M'sila s'est urbanisée de six (06) lotissements et sept (07) cités collectives. Les lotissements promus après 1983 devaient être équipés, selon les directives de la circulaire du 31 mars 1983, d'espaces verts, d'espaces de jeux et de regroupement, dont les superficies devaient être proportionnelles au nombre d'habitant du lotissement $\left(2 \mathrm{~m}^{2} \mathrm{~d}\right.$ 'espace vert/habitant). Or, ni sur le plan théorique (parcellaire), ni sur le plan pratique (l'état réel de ces configurations spatio-urbaines) cette norme n'a été respectée. De même les espaces verts des cités collectives avaient subis le même sort que ceux des lotissements, dans la mesure qu'ils n'avaient de juste que le nom. En effet, loin de constituer des espaces végétalisés, ils formaient plutôt des espaces résiduels de prospect abandonnés

Si durant la décennie 1979-1989, l'espace vert avait largement augmenté en termes de nombreil avait, malheureusement, connu une nette diminution durant la décennie 1989-1999, et ce, malgré les directives de la circulaire du 31 octobre 1984 qui incitait à plus de création en synergie avec l'urbanisation. D'autre part, l'on remarque que le ratio espace vert/ habitant (EV/H) n'a jamais fait 
l'objet d'une volonté ni du concepteur, ni du promoteur, ni du contrôle des services publics concernés. La courbe de ce ratio, montre les pics variant de $9,60 \mathrm{~m}^{2} / \mathrm{h}$, comme plus haut ratio, à $0,26 \mathrm{~m}^{2} / \mathrm{h}$, comme plus faible, en passant parfois par le zéro $(0) \mathrm{m}^{2} / \mathrm{h}$ comme cas le plus défavorable.

Pendant les deux décennies 1979-89 et 1989-99, la moyenne des ratios (EV/H) avait enregistré $1,16 \mathrm{~m}^{2} / \mathrm{h}$. Cependant la moyenne de chaque décennie a été enregistré de l'ordre de 1,47 à $0.84 \mathrm{~m}^{2} / \mathrm{h}$. Soit une régression de l'ordre de $0.63 \mathrm{~m}^{2} / \mathrm{h}$ dans une décennie, (Voir Figure 5 ). Cela révèle le degré de délaissement des acteurs de la ville à ces espaces d'intérêts publics.

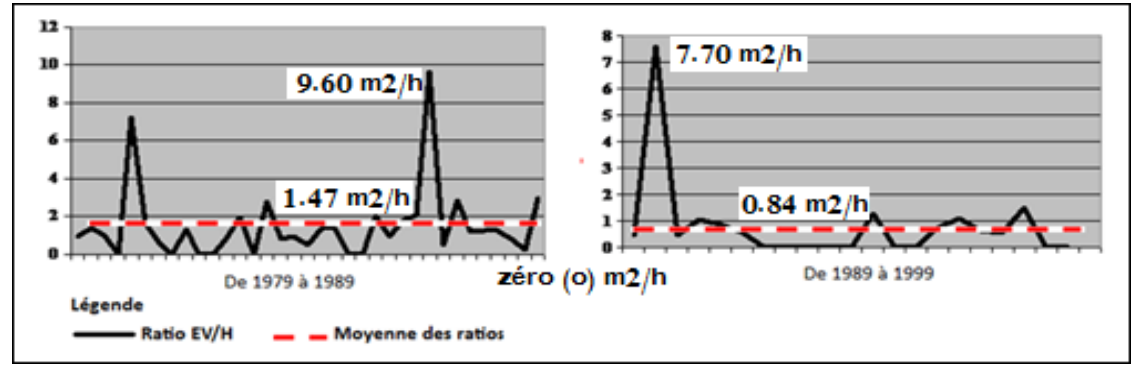

Figure 5 - Évolution du ratio espace vert / habitant des cités d'habitations durant les décennies 1970 et 1980 Source: Mili et Boutabba, 2013.

La période après l'indépendance s'est caractérisée par une nette réduction du "cadre vert" agressé par l'envahissement du "cadre bâti" accompagné d'une structure viaire importante. Nous avons aussi souligné un déséquilibre en matière du nombre de chaque catégorie d'espace vert. Malgré la conception de plus de 32 hectares d'espaces verts au niveau des plans de masse, M'sila ne comporte que 10,61 ha d'espaces verts réellement aménagés (Voir Tableau 2). Non seulement, la superficie de ces espaces est insuffisante par rapport à la norme, mais leurs surfaces aménagées est largement inférieure à celle théoriquement conçue.

Tableau 2 - Superficie des espaces verts existants comparée aux plans de masse et à la norme

\begin{tabular}{cccccccc}
\hline $\begin{array}{c}\text { Zone } \\
\text { urbaine } \\
\text { secteur }\end{array}$ & $\begin{array}{c}\text { Superficie } \\
\text { foncière en } \\
\mathbf{( H a )}\end{array}$ & $\begin{array}{c}\text { Surface } \\
\text { libre en } \\
\mathbf{( H a )}\end{array}$ & $\begin{array}{c}\text { Surface } \\
\text { espaces } \\
\text { verts } \\
\text { urbains }\end{array}$ & $\begin{array}{c}\text { Surface } \\
\text { jardins } \\
\text { privés }\end{array}$ & $\begin{array}{c}\text { Population du } \\
\text { secteur }\end{array}$ & $\begin{array}{c}\text { Ratio 1 } \\
\text { EV.Urbains } \\
\text { par Habitant }\end{array}$ & $\begin{array}{c}\text { Ratio 2 } \\
\text { EV.Jardins } \\
\text { privés par } \\
\text { Habitant }\end{array}$ \\
\hline 1 & 317 & 0 & 0 & 154 & 22.874 & 0 & 67.33 \\
2 & 240 & 0 & 6.8 & 250 & 31.100 & 2.19 & 80.38 \\
3 & 172 & 12.25 & 0.92 & 0 & 24.817 & 0.36 & 0 \\
4 & 168 & 80 & 0.28 & 0 & 10.008 & 0.28 & 0 \\
5 & 323 & 116 & 1.36 & 0 & 4.775 & 2.85 & 0 \\
6 & 271 & 88.90 & 1.25 & 0 & 7.272 & 1.72 & 0 \\
7 & 280 & 0 & 0 & 0 & 0 & 0 & 0 \\
Total 1 & 1771 & 304 & 10.61 & 290 & 100.846 & 1.05 & 28.75 \\
Différents & 729 & 519.60 & 2.09 & -140 & 89.456 & 0.24 & $/$ \\
plans POS* & 2500 & 823.60 & 12.70 & 150 & 190.302 & 0.66 & 7.88 \\
Total 2 & & & & & & & 0 \\
\hline
\end{tabular}

*POS: Plan d'occupation du sol révisé en 2010. Source: Mili et Boutabba, 2013.

En 1966, lors du premier recensement de l'Algérie indépendante, la population M'silienne comptait 22.874 habitants répartie sur une superficie urbaine de 154 hectares (Voir Figure 6). Par conséquent, la part d'espace verdoyant par habitant était de $67,33 \mathrm{~m}^{2}$. Si nous considérons les terrains périurbains réservés à la culture maraichère de l'ordre de 2367 ha, le ratio atteint 1282,50 m2 d'espaces verts par habitant. Or, ceux-ci ont nettement diminué pour atteindre en 1997 le ratio de 1,62 m2/h (Mimoune, 1999). En 2001, le ratio était de $1,05 \mathrm{~m}^{2}$ par habitant (Mili \& Boutabba, 2013). Actuellement, avec l'urbanisation intensive de la ville, le ratio est devenu alarmant tout en atteignant le chiffre de $0,66 \mathrm{~m} 2 / \mathrm{h}$ (Voir Figure 7). 


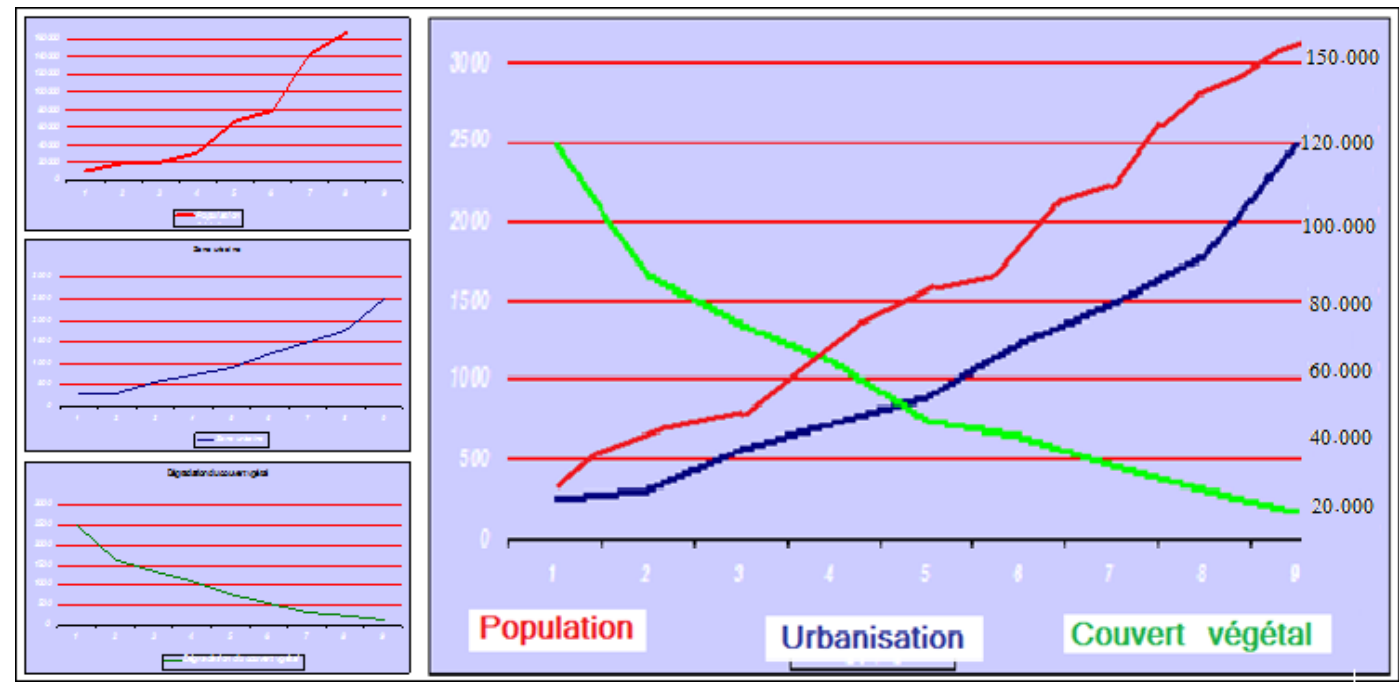

Figure 6 - Évolution de la population, urbanisation et ratio des espaces verts urbains de la ville de M'sila. Source: Mili, 2018.

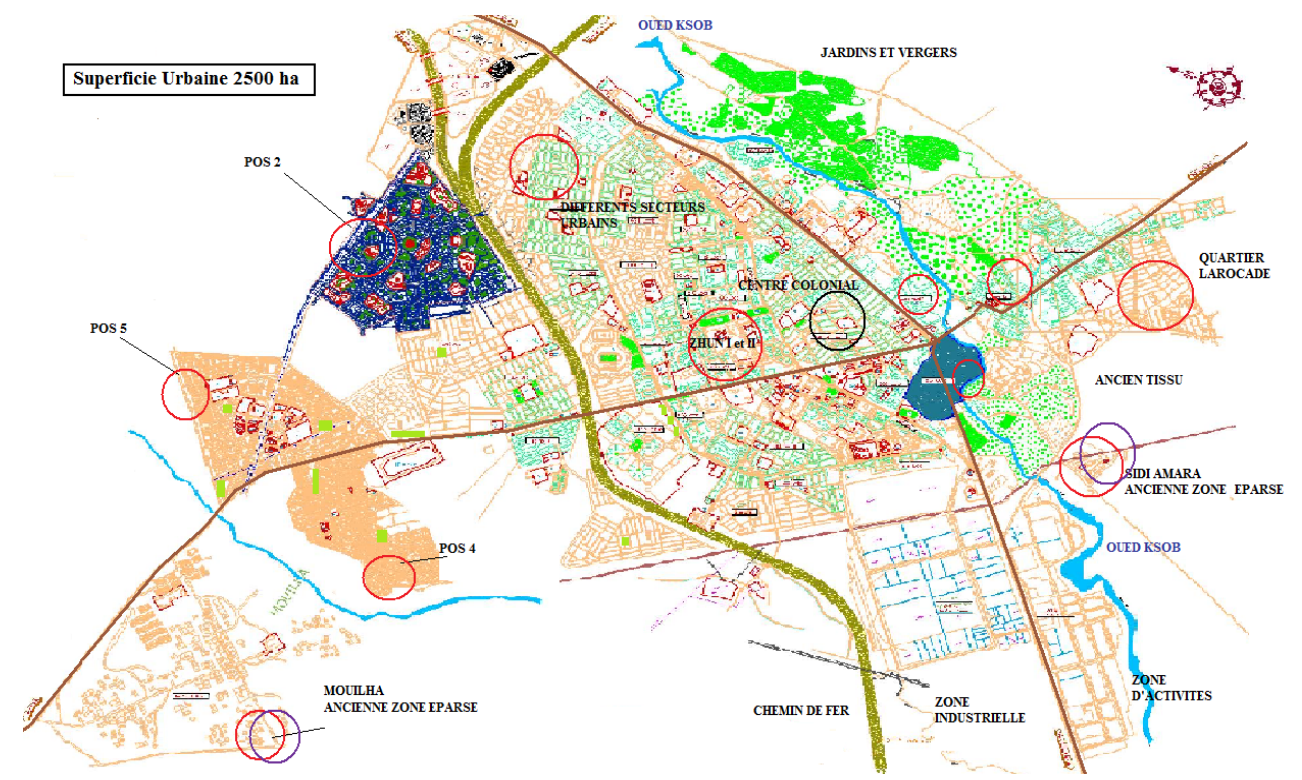

Figure 7 - Étalement urbain de la ville de M'sila. Source: Plan directeur d'aménagement et d'urbanisme PDAU, 2010.

\section{Diagnostic des différentes catégories d'espaces verts de la ville de M'sila}

Le diagnostic va s'intéressé à cinq catégories d'espaces verts: Les arbres d'alignement urbain, les espaces verts de proximité des cités d'habitations collectives, ceux des lotissements, Jardins publics et squares.

\section{Arbres d'alignement urbain}

En plus du rôle esthétique incontestable qu'ils procurent, les arbres d'alignement assurent également un micro climat bénéfique à l'homme, ils créent une harmonie et une liaison entre voirie et construction, réduisent la propagation sonore et allègent la pollution de l'air. Actuellement, dans la ville de M'sila, les arbres d'alignement sont principalement limités aux bordures des grandes voies et aux axes 
principaux de la ville, notamment le long de la partie Ouest de l'axe Rocade-Ichebilia, et l'axe Nord-sud: Bordj BouArréridj- Boussaâda (25\%). Ils diminuent au niveau des boulevards (10\%) et sont presque absents au niveau des petites voies secondaires (5\%). Quant aux doubles voies, on remarque la plantation de palmiers "Washingtonia", d'arbustes et d'arbrisseaux, comme type de plantation des terre-pleins (Voir Figure 8).
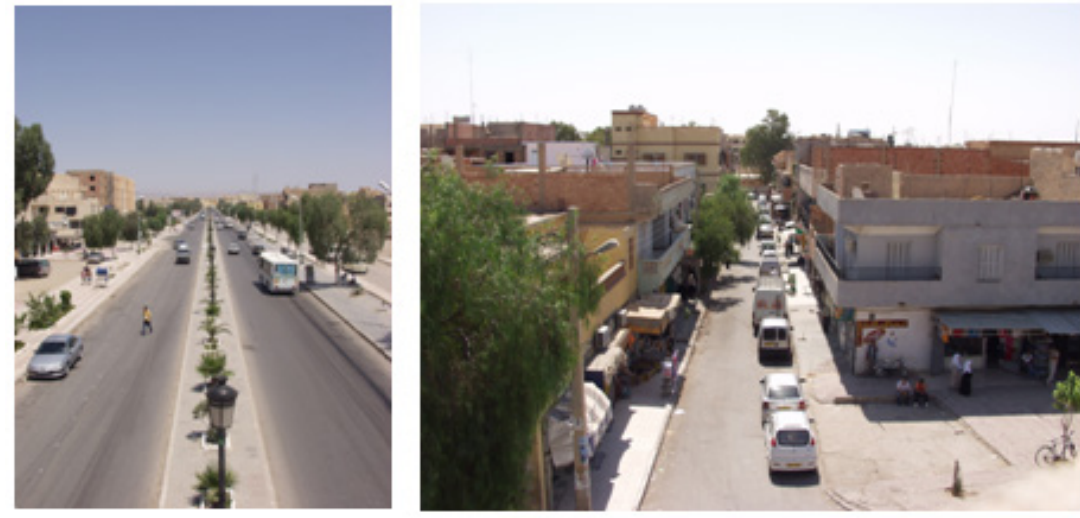

Figure 8 - Plantation des arbres d'alignement urbain. Source: Auteurs, cliché 2010.

En général les arbres d'alignement de la ville de M'sila présentent un état sanitaire moyen. L'espèce végétale le plus utilisée est le palmier et le faux poivrier qui s'adaptent bien au climat semi-aride de la région et au type du sol. Cependant, le nombre reste insuffisant. Ces alignements essayent, tant bien que mal, de procurer de l'ombre aux piétons et de contribuer à l'organisation de la circulation. Quant à l'aspect esthétique, ces alignements contribuent certainement à l'embellissement de la ville, mais vu leur nombre réduit, le manque de traitement phytosanitaire ainsi que l'élagage, beaucoup d'actions restent à développer.

D’après la subdivision des travaux publics de la wilaya de M'sila (Algérie, 2013), la voirie urbaine de la ville est évaluée à $260 \mathrm{Km}$. Les besoins en arbres d'alignement pour planter toutes les voies urbaines de part et d'autre, suivant la circulaire du 31 octobre 1984 (Algérie, 1984), est de 104.000 arbres, alors que la ville n'en dispose que d'environs 20\%, (Conservation des Forêts, 2015). Cependant, il ne suffit pas d'avoir la volonté de planter, mais plutôt de bâtir en adéquation avec l'espace vert urbain. Le volume du cadre bâti des habitations ne doit pas entraver l'évolution de l'arbre qui se trouve dans les vérandas des maisons, de même le trottoir doit avoir une largeur adéquate permettant d'accueillir ces arbres (voir Figure 9).
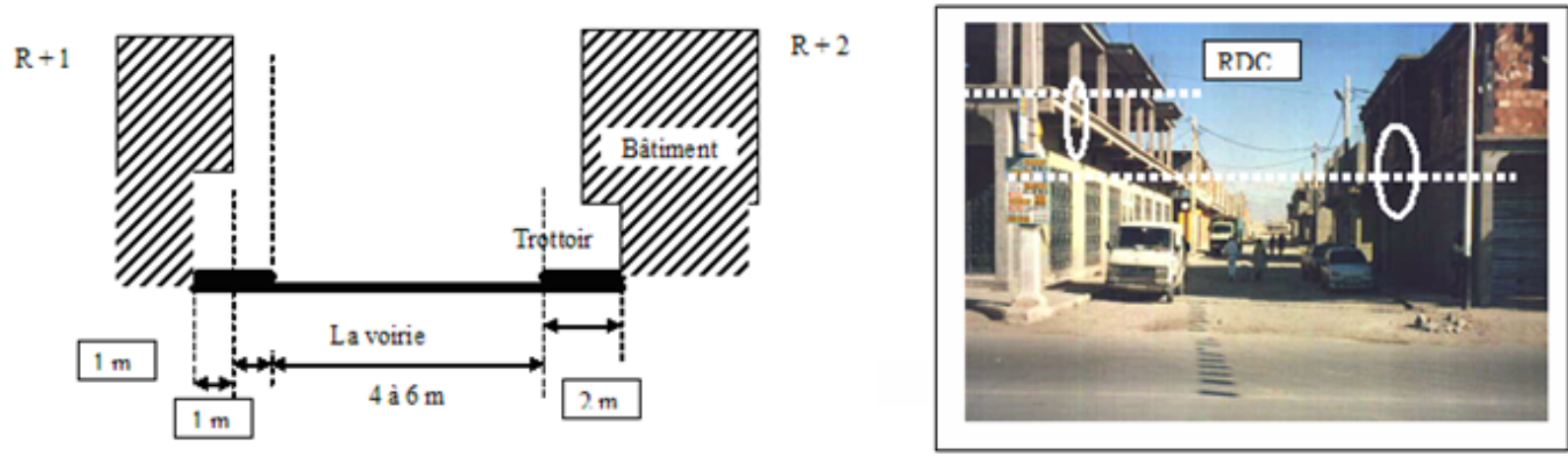

Figure 9 - Coupe transversale sur voie secondaire: arbre et cadre bâti. Source: Mili (2002).

La Figure 9 montre clairement que le volume qui résulte d'une telle pratique conceptuelle ne permet en aucun cas, la possibilité de planter des arbres d'alignement ni même d'arbustes. La plantation de quelques haies dont la hauteur ne doit pas dépasser les deux mètres de haut semblent présenter la 
seule alternative. La probabilité de planter un arbre dans une telle conception de trottoir est à éliminer, non seulement à cause de la hauteur du cadre bâti et du balcon en porta-faux, mais aussi de la largeur réduite du trottoir ( 1 à $2 \mathrm{~m}^{2}$ ). Le cas le plus déplorable est celui de la cité 500 logements Mankoubine où des arbres centenaires risqueraient d'être sacrifiées vu les extensions horizontales et verticales du bâti suite à la création de galeries piétonnes au niveau des grands boulevards (Voir Figure 10).

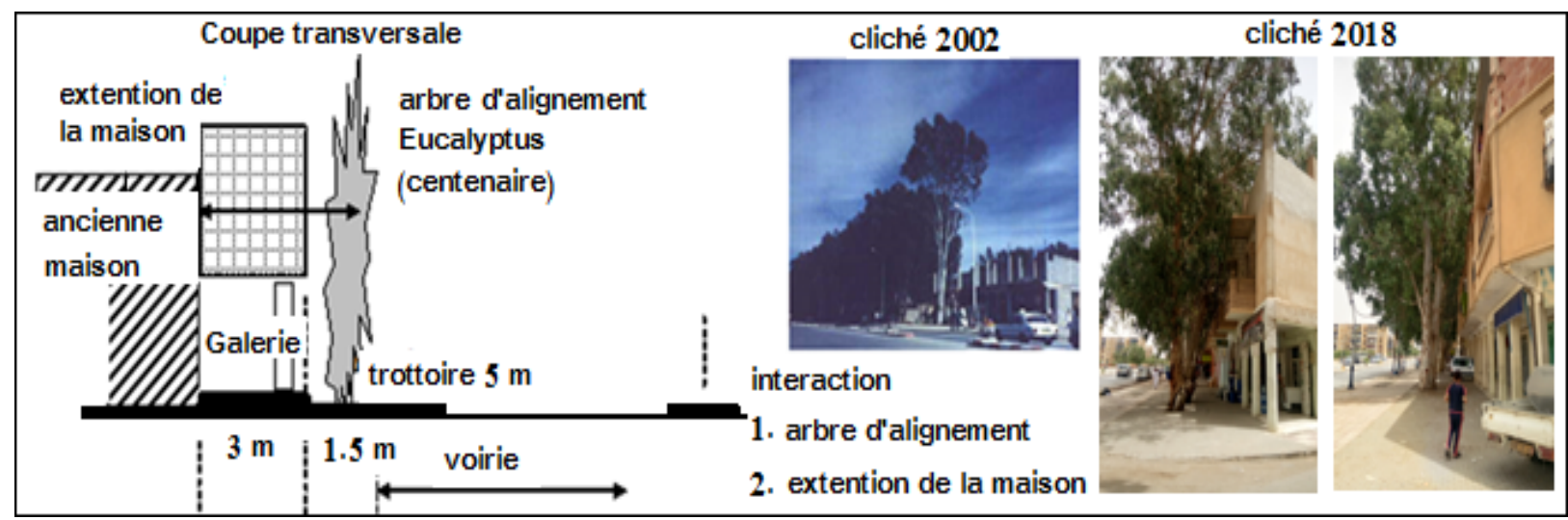

Figure 10 - Une extension autorisée du cadre bâti au détriment des arbres d'alignement centenaires. Source: Mili (2002).

\section{Les espaces verts de proximité des cités d'habitations collectives}

La promotion de la daïra de M'sila en wilaya s'est traduit par la naissance de plusieurs cités collectives. Ces cités sont constituées d'un grand nombre d'immeubles identiques et répétitifs engendrant un environnement terne, monotone et anonyme. Cette monotonie urbaine provient d'un manque flagrant d'aménagement des espaces extérieurs notamment l'espace vert (Zucchelli, 1984). Em effet, lors de l'implantation des bâtiments, le souci majeur des concepteurs était d'organiser les blocs selon un principe conceptuel bien déterminé (bloc barre, bloc tour) en omettant une réflexion sérieuse de l'espace vert. Si dans le pays européens notamment en France, la construction des grands ensembles durant la décennie des années 1970 constituait un tournant crucial dans les politiques de planification et d'aménagement des espaces verts (Reygrobellet, 2007), en Algérie depuis les années 1970 et jusqu'à présent, l'espace vert de ce genre d'opération urbaine n'a jamais fait l'objet d'une étude préalable prenant en compte les facteurs climatiques sociaux et urbains, mais se limitait à un espace résiduel non réfléchi et non hiérarchisé (Mezrag, 2015). Les enseignements du Mouvement Moderne du début du 20e siècle vis à vis de l'aménagement des espaces verts (Cunha, 2009) semblent encore d'actualité. Fondamentalement, cet état est l'image de la plus grande majorité des cités collectives de la ville qui ne diffère pas l'une de l'autre.

\section{Les espaces verts des lotissements}

Devenant le principal promoteur immobilier, l'agence foncière a finit par promouvoir dès 1979 neuf (09) lotissements. La ville de M'sila avoisine les 69 lotissements soit plus de 19.000 lots à construire. Em plus des îlots, ces lotissements devraient être constitués selon la réglementation en vigueur, d'aménagements extérieurs, constitués entre autres, par des espaces verts (Loi $n^{\circ}$ 90-29, Algérie, 1990b), autrement dit le dossier graphique joint à la demande du permis de lotir doit contenir d'après l'article 09, la localisation des espaces verts.

Officiellement, le lotissement doit être constituéd'un certain nombre de lots regroupés en îlots, séparés par des voiries. Pour permettre une certaine vie sociale et communautaire entre les co-lotis, le 
lotissement doit prévoir des espaces de regroupements, des aires de jeux et des espaces verts. Ces espaces verts doivent avoir une surface qui représente soit $2 \mathrm{~m}^{2}$ par habitants (circulaire du 31 octobre 1984) soit $6,8 \mathrm{~m}^{2}$ par habitant (circulaire interministérielle du 31 octobre 1984). Or réellement cette norme est loin d'être respectée. Le phénomène peut-être expliqué par ce qui suit: Au début des années 1990 et suite à la croissance démographique qui a caractérisé la ville de M'sila d'une part, ainsi que son industrialisation et son urbanisation d'autre part, le principal promoteur de lotissement, l'agence foncière était obligée de créer un nombre important de lots résidentiels. Or la crise économique difficile qui s'est manifestée dans toute son acuité, durant la décennie des années 2000 ainsi que la saturation des terrains, ont favorisé non pas la création de nouveaux lotissements, mais plutôt la densification des anciens lotissements. Ainsi le coût des travaux des voiries et réseaux divers se trouve nettement diminué.

Cette densification se faisait au détriment des assiettes foncières réservées aux équipements et dans la plus part des cas au détriment des terrains réservés aux espaces verts. La surface affectée aux espaces verts peut facilement changer de vocation pour devenir des lots résidentiels. Même si cette densification touche l'ensemble des réserves foncières, elle est très présente et très ressentie au niveau des lotissements. Il s'est même avéré, d'après l'étude de Boutabba (2001) que dans la majorité des cas, les promoteurs font exprès de ne pas publier les actes de leurs lotissements auprès de la direction des domaines pour faciliter le changement de vocation de ces poches foncières d'intérêts publics. Ces poches sont plein d'enjeux. En l'absence d'un organisme bien identifié par la réglementation urbaine, voué à la protection de l'espace vert, ce "parent pauvre" de la ville, avait longtemps vu sa superficie diminuée d'une année à l'autre. Cependant depuis la promulgation de la loi n07-06 du 13 mai 2007 (Algérie, 2007), relative à la gestion, à la protection et au développement des espaces verts dans le cadre du développement durable, une amélioration bien qu'encore timide et une nouvelle vision sur la valorisation de l'aspect vert dans le paysage urbain notamment celui des lotissements commence à être ressentie.

\section{Jardins publics et squares}

Dans la présente étude le square est entendu dans son acceptation française et non londonienne ${ }^{2}$ comme «un jardin public formé au centre d'une place bordée de façades, contourné par les circulations » (Merlin \& Choay, 1996). La ville de M'sila souffre d'un manque flagrant en matière de squares et de jardins publics. En effet elle ne compte que deux jardins proprement dit: Le jardin 'Ahmed Madeghri' et la place '1er novembre 1954' (Voir Figure 11). L'ensemble de ces jardins constitue les composantes les plus représentatives de l'espace vert à M'sila. Le jardin 'Ahmed Madeghri' était, jusqu'à la décennie des années 2000 , totalement clôturé. Il couvre une superficie étroite et une géométrie simple, basée sur la centralité où le coté minéral est entrain de prendre, par les nouveaux aménagements, le dessus sur le coté végétal. La place '1er novembre', malgré qu'elle soit ouverte au public, dépourvue de clôture, vaste comparativement aux autres squares et placettes de la ville, ne constitue qu'un espace de transition, sans aucune activité sportive ou de détente. Cette place souffre comme dans la plus part des cas des espaces végétalisés de la ville, d'un manque de variété textuelle accentué par une pauvreté de couleurs, ce qui la rend terne et monotone. Cette placette souffre aussi d'insuffisance et d'inopérationnalité des points d'eaux que ce soit jet d'eau, fontaine ou bassin, malgré leur importance dans les climats semiarides tel que celui de la ville de M'sila. Quant à la végétation, qui devra constituer un élément fondamental pour rehausser les qualités visuelles et qualités du confort urbain, elle n'est constituée dans la quasi-totalité des jardins, squares et autres espaces verts que d'arbres et d'arbustes sans aucune variété des espèces végétales et des essences. On dénote un manque de gazon, de fleurs et de plantes

2D'après Merlin \& Choay (1996) le square londonien, espace libre au centre d'un îlot quadrangulaire, réservé à l'usage des riverains, à l'origine espace minéral servant de cour qui fut ensuite souvent planté. 
grimpantes. Le sol de ces espaces, au lieu d'être formée de pelouse ou à la rigueur de haie végétale, il est constitué par des matériaux artificiels tels que l'asphalte, le ciment et parfois de pavés en marbre. Le mobilier urbain ne semble pas faire l'exception à cet état général déplorable. Il est composé exclusivement de banquettes en béton à l'état dégradé et quelques fois des lampadaires généralement non fonctionnels.
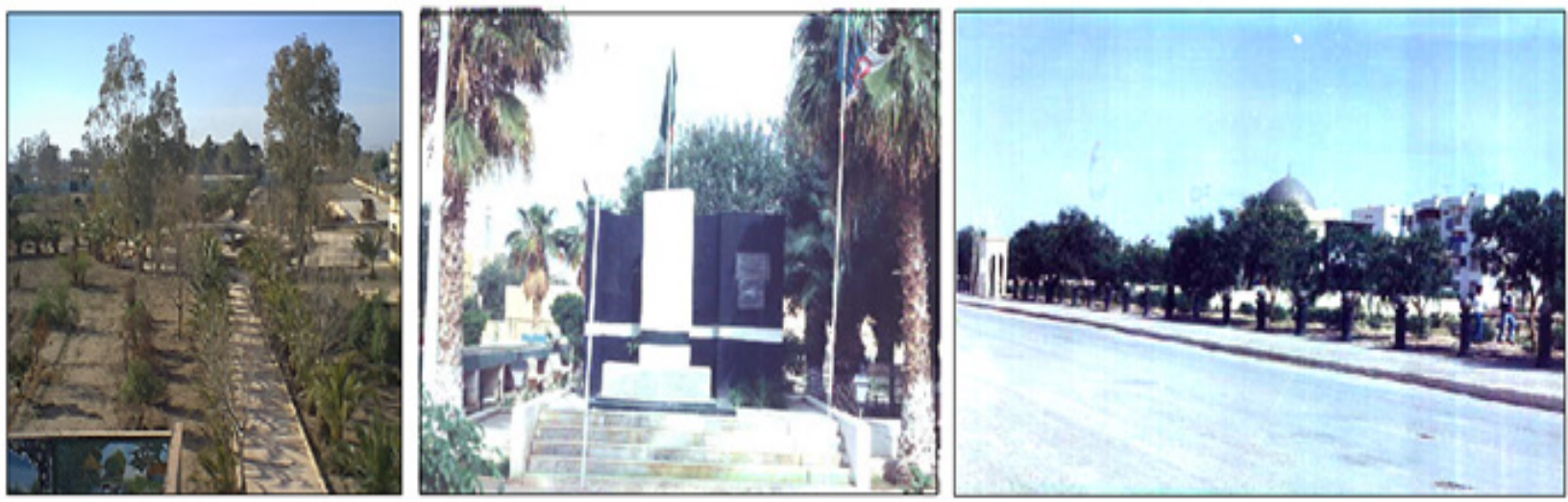

Figure 11 - Parc et jardins publics de la ville de M'sila. Source: Mili, clichés 2002.

\section{Interaction espace vert, gestionnaires de la ville}

Le végétal est non seulement un outil d'amélioration du cadre de vie ou un simple élément marquant de notre quotidien, mais aussi un élément vivant (Voir Figure 12) qui nécessite un entretien et une gestion durant toute sa période de croissance, jeunesse (cinq ans), maturité et vieillissement.

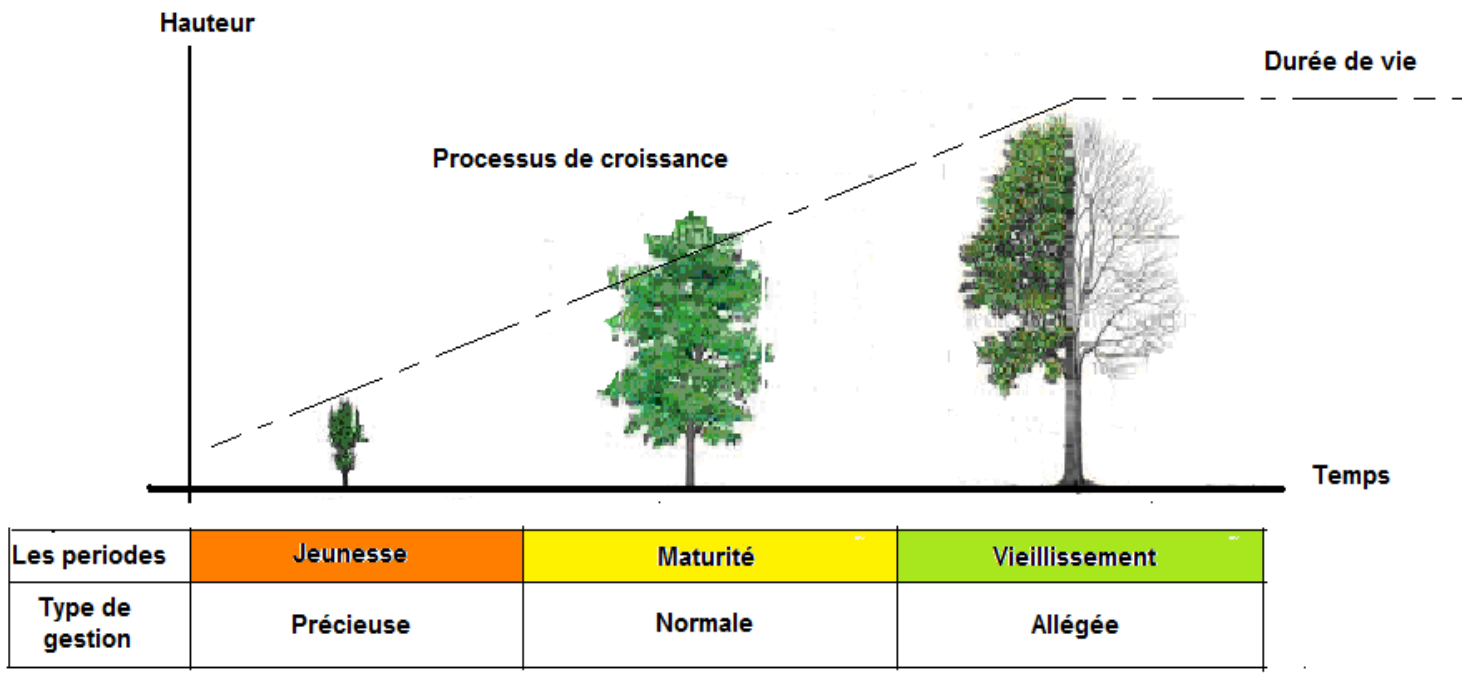

Figure 12 - Le processus de croissance d'un espace vert. Source: Mili (2002).

L'espace vert n'est pas une surface à créer ou à aménager puis abandonner dès la fin de sa création, bien au contraire la naissance d'un espace vert suscite son éducation dans le sens large du mot, autrement dit l'entretenir à vie en assurant la bonne croissance des végétaux, sa bonne santé, sa vitalité et tirer le profit des floraisons espérées.

Un habitant (enfant ou adulte) en cassant les branches d'un arbre ou en le coupant, pratique un acte de dégradation. Un gestionnaire de la ville qui abandonne un espace vert, n'arrose pas un arbre, ne respect pas les normes ou autorise une densification au détriment des espaces verts, favorise aussi une 
pratique qualifie d'acte de dégradation. Cela nécessite une réflexion et une correction à tous les niveaux d'action des acteurs de la ville pour mieux promouvoir l'espace vert.

La ville de M'sila est dotée de certaines directions qui devraient, d'une façon directe ou indirecte, entretenir les espaces verts de la ville, à savoir: Direction technique de l'assemblée populaire communale (APC), la conservation des forêts et la direction de l'environnement.

\section{La direction technique de l'APC de M'sila}

En Algérie, l'aménagement et la gestion urbaine de la ville (toutes les actions confondues) sont légués au secteur étatique notamment à l'APC. Les services techniques de cette dernière procèdent à l'aménagement et à la gestion urbaine selon les priorités d'urgence. La direction technique de l'APC est structurée selon deux services. Le service construction et urbanisme qui s'occupe du suivi du cadre bâti et de l'aménagement extérieur et le service matériel et maintenance qui s'occupe du nettoyage urbain, de la collecte des déchets et de l'entretien des espaces verts. Ce service attribue cette tache, sans aucune stratégie d'intervention, à de simples agents non qualifiés. A M'sila, Seuls trois ouvriers permanent s'occupent de la gestion de tous les espaces verts de la ville. Cette gestion se limite aux simples travaux d'arrosage, d'élagage des plantes et parfois de plantation. En cas de nécessité, le service peut demander un recrutement, qui se fait généralement, par l'emploi de jeunes, par voie de contrat à durée limitée, sans se préoccuper de leurs qualifications. Le nombre de ces recrutements n'a jamais excédé une dizaine. Les moyens mis à la disposition de ces équipes temporaires sont dérisoires et se limitent à un petit outillage, trois citernes d'eaux pour l'arrosage mobile et un véhicule de service. Ces équipes s'auto organisent avec les moyens disponibles pour intervenir dans l'entretien notamment l'arrosage du plus grand nombre possible d'espaces verts sans pouvoir respecter le cycle d'irrigation.

\section{La direction de conservation des forêts}

La direction de conservation des forêts est un organisme étatique, il a pour mission de gérer tout le patrimoine forestier des régions urbaines et périurbaines en assurant la protection de son composant naturel: l'arbre. En termes de gestion cet impératif implique la promotion et l'intensification des espaces verts et de leurs sauvegardes de toutes actions de détournement de leurs vocations. Les agents de la conservation des forets sont habilités conformément aux dispositions législatives en vigueur à procéder à des sanctions judiciaires à l'encontre des contrevenants ayant abattu un arbre ou contribué d'une façon directe ou indirecte à la dégradation d'un espace vert. Or depuis la création, de cette direction aucun procès verbal n'a été dressé, et ce, en dépit des détournements de vocation d'un nombre important d'espaces verts par l'agence foncière de M'sila, premier lotisseur étatique de la ville.

\section{La direction de l'environnement}

La direction de l'environnement est responsabilisée, suite a la loi nº7/06 (Algérie, 2007), au même titre que la conservation des forets, à protéger l'environnement d'une façon générale et l'arbre d'une façon particulière et ce pour les différentes catégories d'espaces verts. La direction de l'environnement est autorisée à prendre toutes les dispositions nécessaires en faveur de la protection des espaces verts et des espaces boisés qui représentent un patrimoine naturel et ce dans le but d'améliorer le cadre de vie des habitants, de promouvoir la création d'espaces verts dans les milieux urbains, de veiller au respect et l'application des sanctions en cas de dépassements formulés dans la loi relative à la protection de l'environnement.

A ce jour la participation de cette direction en matière de conservation du cadre vert se limite à l'établissement des bases de données, des statistiques et des rapports sur l'état des lieux, du fait qu'ils 
seront communiqués au ministère pour exploitation. Actuellement il est demandé à la direction d'évaluer les besoins de la commune de M'sila en matière d'espaces verts à l'intérieure des établissements scolaires et de préparer un devis quantitatif et estimatif pour une éventuelle intervention publique en coordination avec la direction de l'éducation et de l'enseignement.

\section{Les organisations associatives}

Les organisations associatives jouent un rôle important dans la protection et le maintien des espaces verts. Leur influence sur le comportement des habitants envers les espaces verts est remarquable. Une fois organisés sous forme de groupes associatifs, les habitants procèdent à la mise en place d'une stratégie d'action dans le but d'améliorer leur cadre de vie et la qualité de leur environnement. C'est ainsi que la gestion des espaces verts est concertée entre services municipaux et habitants par l'intermédiaire des organisations associatives. Ces dernières représentent, pour les acteurs de la ville, une source d'informations et de sensibilisation, comme elles représentent pour les habitants un moyen de revendication. Le degré d'appropriation des espaces verts par les habitants a une incidence directe sur le coût de gestion de ceux-ci, dans la mesure où un public sensibilisé est un public éduqué et motivé et par conséquent, au lieu de dégrader l'espace vert, il contribue à sa promotion. Malheureusement, il est à constaté que les associations autorisées à exercer dans la ville de M'sila ne se préoccupent que des problèmes relatifs à la dégradation des réseaux d'assainissements d'eau usée (AEU) et d'eau potable (AEP) dans leurs quartiers résidentiels. Par négligence ou par ignorance, l'aménagement et l'entretien des espaces verts sont totalement absents de l'ordre des priorités.

\section{Les entreprises privées}

La wilaya de M'sila, principalement agro-pastorale, compte selon les statistiques de la direction de l'administration locale DAL, une quarantaine (40) d'entreprises privées spécialisées dans l'exploitation forestière et la gestion des espaces verts urbains et périurbains. Sauf que, seulement douze (12) d'entre elles sont qualifiées. La majorité restante de ces entreprises ne détient pas de certificat de qualification puisqu'elles manquent de personnel technique et scientifique spécialisé en agronomie, géologie et aménagement urbain. Le maitre d'ouvrage principal qui offre du travail à ces entreprises reste la conservation des forêts (organisme étatique). La quasi-majorité de ses projets concerne la réhabilitation des exploitations forestières dans tout le territoire de la wilaya de M'sila estimé à $6.951 \mathrm{~km} 2$ (Conservation des Forêts, 2015).

Durant les dernières années, les collectivités locales ont reçu des instructions, de la part de la tutelle (Ministère de l'intérieur, des collectivités locales et de l'aménagement du territoire MICLAT) de réduire leur gestion de régie des espaces verts, notamment urbains et publics, tout en impliquant les prestataires privés. A travers cette stratégie, la commune de M'sila, en coordination avec les secteurs socio-économiques, avait établi un plan de gestion organisé par strates. Ce plan de gestion consiste à passer des conventions et des marchés avec les entreprises privées pour améliorer l'entretien des espaces verts selon leurs caractéristiques et leurs classifications. Pour le secteur public la procédure de passation des conventions et marchés se fait conformément au décret présidentiel $n^{\circ} 02 / 250$ du 24 juillet 2002, modifié et complété par le décret présidentiel n 15/247 du 16 septembre 2015, portant réglementation des marchés publics et des délégations de service public (Algérie, 2002). Théoriquement, le rôle des entreprises dans l'aménagement, entretien et gestion des espaces verts est prospérant, mais en pratique, il reste très limité. La rareté des subventions budgétaires publiques allouées à la gestion des espaces verts et la pénurie des ressources en eau dans le milieu steppique constituent les principales contraintes qui entravent la mise en œuvre du plan de gestion. 


\section{Evolution des politiques publiques en matière d'espaces verts urbains}

Le développement des potentialités quantitatives et qualitatives des espaces verts urbains est assujetti à plusieurs facteurs dont ceux liés à la réglementation et aux stratégies urbaines adoptées (Voir Tableau 3).

Nous rappelons que durant la période de la colonisation (1830 - 1962) l'Algérie était régie par la réglementation Française. De ce fait, l'avènement du 20é siècle s'était annoncé par un renouveau en matière des espaces verts localisés dans les agglomérations urbaines. Ce renouveau s'est traduit des l'année 1901 par la Loi relative à la création des activités associatives qui a favorisé la naissance d'un organisme nommé "service des cultures" structuré par deux divisions. L'une décorative, axée sur la protection et l'entretien des végétaux dans le milieu urbain. L'autre scientifique, axée sur l'étude et le développement des espaces végétales. Depuis, plusieurs textes législatifs et dispositifs d'aménagement urbains se sont succédés afin d'améliorer, de créer et de préserver les espaces verts. La promulgation de la Loi Cornudet en 1919 a permis aux communes d'établir des plans d'extension et d'embellissement des différentes catégories d'espaces verts dans les villes (France, 1919). La Loi Prothin promulguée en 1943 a intégré la notion de zonage (habitat et espaces verts) pour rationaliser l'utilisation des sols à urbaniser (France, 1943).

A l'aube de l'indépendance (1962), certaines réglementations héritées de l'administration Française sont resté appliquées jusqu'à l'adoption de la charte nationale en 1976 (Algérie, 1976). La priorité de l'État Algérien indépendant était focalisée sur le secteur de l'éducation, de l'agriculture et des hydrocarbures. La gestion des espaces verts, considéré à l'époque comme un besoin social de luxe, était reléguée au dernier rang des priorités. Durant les années quatre-vingt (1980), la circulaire interministérielle du 31 Octobre 1984 relative aux normes des espaces verts dans les zones urbaines avait imposé l'augmentation du ratio de $2 \mathrm{~m} 2$ à $6,8 \mathrm{~m} 2$ d'espaces verts par habitant dans les cités résidentielles. La circulaire avait incité les acteurs urbains à créer des espaces verts en synergie avec l'urbanisation. Après constatation des pouvoirs publics sur le degré de dégradation des espaces verts, La Loi 83/03 du 5 janvier 1983 relative à la protection de l'environnement est venue définir la sauvegarde de l'environnement comme étant une exigence fondamentale de la politique nationale de développement économique et social (Algérie, 1983). Avec la promulgation de la Loi 90/08 du 7 avril 1990 relative à la commune, les collectivités locales ont été chargées de l'aménagement et la gestion des espaces verts publics (Algérie, 1990a). L'article 108 de la Loi stipule "La commune prend en charge la création et l'entretien des espaces verts et de tout le mobilier urbain visant à l'amélioration du cadre de vie des citoyens." La Loi 90/29 du 01 décembre 1990 relative à l'aménagement et l'urbanisme avait instauré deux instruments d'urbanisme, à savoir, le plan directeur d'aménagement et d'urbanisme PDAU et le plan d'occupation du sol POS (Algérie, 1990b). Le premier, défini les orientations générales d'utilisation des sols et le second, fixe les usages des sols. Ce dernier, une fois publié, est imposable aux tiers.

Dans la politique du développement durable, la Loi 07/06 du 31 mai 2007 relative à la gestion, à la protection et au développement des espaces verts a été promulguée afin d'entretenir et d'améliorer la qualité des espaces verts urbains existants et de promouvoir leur création (Algérie, 2007). Cette loi avait imposé deux outils de gestion. Le premier, concerne la classification des espaces verts selon un inventaire préétabli. Le second, concerne l'établissement d'un plan de gestion des espaces verts. La Loi 06/06 du 20 février 2006 portant loi d'orientation de la ville s'inscrit dans le cadre de la politique de la ville (Algérie, 2006). Cette politique vise à orienter et à coordonner toutes les interventions publiques notamment dans les domaines de la résorption de l'habitat précaire, de la santé publique et de la protection de l'environnement. 
Tableau 3 - Tableau synoptique des politiques publiques urbaines adoptées

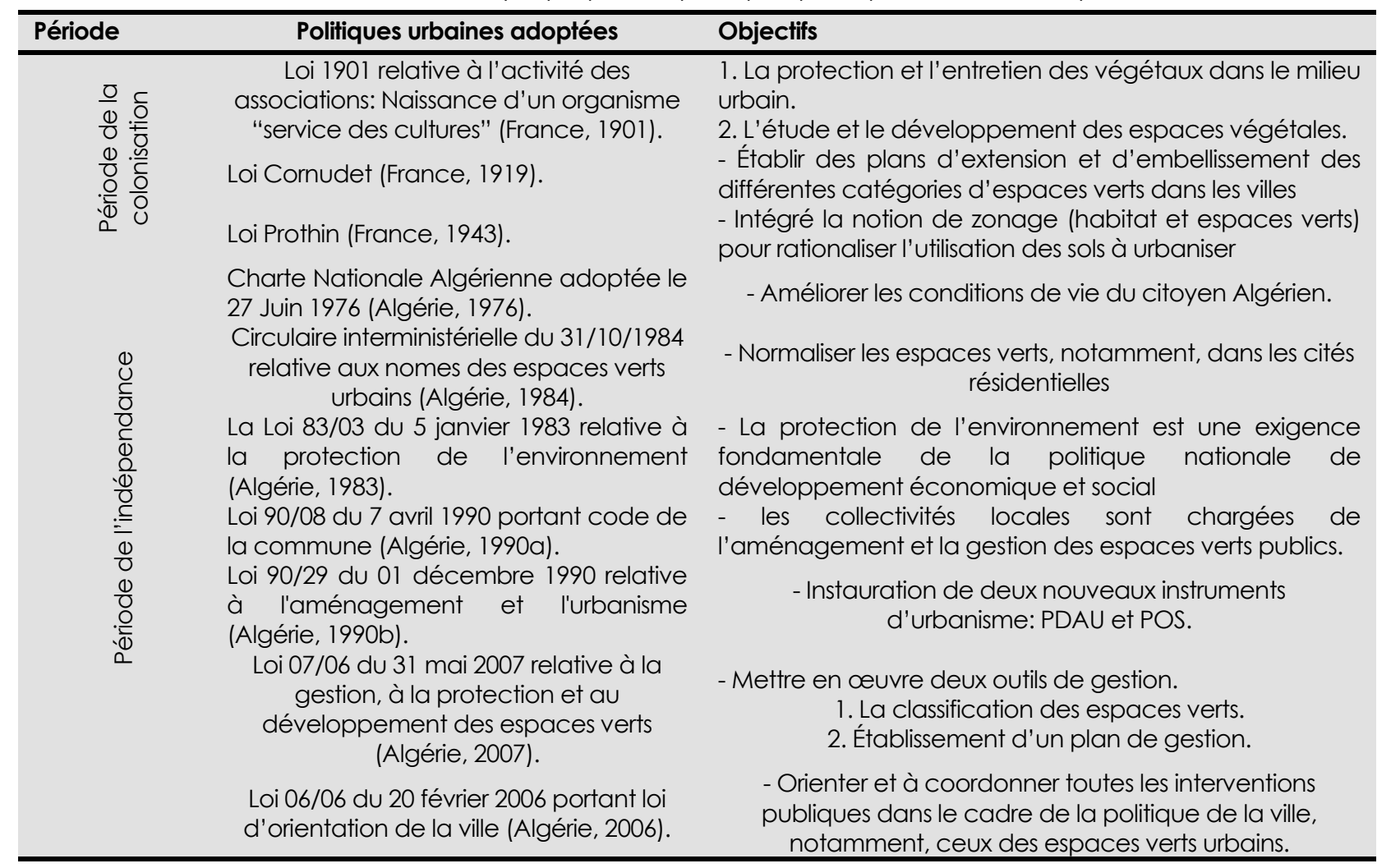

Source: Auteurs, 2018.

\section{Conclusion}

La ville algérienne spécifiquement celle des hauts plateaux steppiques telle que la ville de M'sila estelle un lieu de nature ? si de part le monde, depuis quelques années, de nombreuses disciplines se sont préposées dans la perspective de traiter cette question. (Machon, 2011; Oueslati et al., 2008; Arrif, 2007; Daniel \& Lecamp, 2004; Donadieu, 2005; Bekouche, 1999), la place attribuée aux espaces verts dans les théories, la planification et les politiques urbaines en Algérie reste bien marginalisée, et ce, malgré l'arsenal des textes législatifs en vigueur. En effet, elle est vue comme un concept élitiste et hors de propos en face de la pauvreté et les inégalités dans la vie quotidienne des habitants et par conséquent fait mauvais ménage avec les conditions sociales et finit par engendrer un conflit d'éthique.

Le cadre vert à Msila est d'une année à l'autre sacrifié au profit de l'extension du cadre bâti par les gestionnaires de la ville sensés garantir sa prospérité, sans que ceci alerte l'opinion publique. De ce fait, le cadre vert constitue le 'parent pauvre' de tout le dispositif d'aménagement urbain. L'entretien et la gestion des espaces verts n'ont jamais fait l'objet d'une prise en charge réfléchie par un organisme spécialisé en la matière. La gestion municipale dont s'est appropriée l'APC de M'sila n'a pas pu montrer son efficacité en matière d'entretien et de gestion de ces espaces du fait de l'inexistence de concertation avec les associations ainsi que la marginalisation des entreprises privées à s'associer aux procédures d'entretiens. Le cadre vert dans la ville steppique des hauts plateaux souffre toujours d'un manque de reconnaissance et de considération. Pour endiguer cet état de fait, il serait judicieux de réfléchir à des mécanismes qui pourraient redéfinir de nouveaux rapports entre les habitants et la nature urbaine. 


\section{Bibliographie}

Al Bakri, A. O. (1913). Description de l'Afrique septentrional, trad de Slane (2e. ed.). Alger: Adolphe Jourdan. Al Idrisi, A. (1866). Description de l'Afrique et de l'Espagne, tard de Dozy et de Goeje (2e. ed.). Paris: Leiden. Al Muqaddasi, A. (1950). Description de l'occident musulman au IVe / Xe siècle. Alger: Carbonel. Algérie. (1976, 27 juin). Charte nationale algérienne. Alger: Journal Officiel. Algérie. (1983, 05 janvier). Loi n03/83 relative à la protection de l'environnement. Alger: Journal Officiel. Algérie. (1984, 31 octobre). Circulaire interministérielle relative aux normes d'espaces verts applicables dans les zones urbaines. Alger: Journal Officiel.

Algérie. (1990a, 07 avril). Loi $n^{\circ} 08 / 90$ portant code de la commune. Alger: Journal Officiel.

Algérie. (1990b, 01 décembre). Loi n90-29 relative à l'aménagement et l'urbanisme. Alger: Journal Officiel.

Algérie. (2002, 24 juillet). Decrét presidentiel $n^{\circ} 02 / 250$ portant reglementation des marchés publics. Alger: Journal Officiel.

Algérie. (2006, 20 fevrier). Loi $n^{\circ 06 / 06}$ portant loi d'orientation de la ville. Alger: Journal Officiel.

Algérie. (2007, 13 mai). Loi $n^{\circ}$ 07-06 relative à la gestion, à la protection et au développement des espaces. Alger: Journal Officiel.

Algérie. (2013, 02 avril). Bulletin de la subdivision. M'sila: Subdivision des Travaux Publics.

Ali-Khodja, A. (2000). Aménagement urbain: la problématique de l'espace vert public dans la ville de Constantine. Science et Technologies, (32), 9-18.

Ali-Khodja, A. (2011). Espace vert public urbain. de l'historicisme a la normativité (Thèse de doctorat). Département d'architecture, Université de Constantine, Algérie.

Almi, S. (2002). Urbanisme et colonisation présence française en Algérie. Paris: Pierre Mardaga.

Arrif, T. (2007). Aménagement urbain: toujours plus de nature. Urbanisme, (352), 20-21.

Becker, H. S. (2002). Les ficelles du métier. Comment conduire sa recherche en sciences sociales. Paris: La Découverte.

Bekouche, A. (1999). Les espaces verts urbains publics, lieux de sociabilité et élément de composition urbaine (Thèse de doctorat). Département D’Architecture, Université des Sciences et de la Technologie d'Oran, Algérie.

Boutabba, H. (2001). Le lotissement résidentiel entre la procédure officielle et la procédure parallèle. Cas de la ville de M'sila (Thèse de magistère). Institut de Gestion des Techniques Urbaines De M'sila, Algérie.

Boutabba, H. (2013). Spécificités spatiales et logiques sociales d'un nouveau type d'habitat domestique du Hodna oriental Le type" Diar Charpenti" (Thèse de doctorat). Département D’architecture de Biskra, Algérie.

Boutabba, H., Farhi, A., \& Mili, M. (2014). Colonial archtectural heritage in the Hodna Region, a vanishing legacy: the case of the city of M'sila, Algeria. L'Année du Maghreb, (10), 269-295.

http://dx.doi.org/10.4000/anneemaghreb.2113.

Collection de l'étoile collection Albert. (2017). Récupéré le 11 mai 2017, de https://www.delcampe.net/fr/cartespostales/afrique/algérie

Conservation des Forêts. (2015). Arbres de M'sila. Alger: La Direction.

Cunha, A. (2009). Introduction: urbanisme végétal et agriurbanisme - La ville entre artifice et nature. Urbia. Urbanisme Végétal et Agriurbanisme, 1-20.

Dahmani, K., \& Moudjari, M. (2013). Praxis d'habitat social. Revers et couronnements. Alger: Office des Publications Universitaires. 
Daniel, H., \& Lecamp, E. (2004). Distribution of three indigenous fern species along a rural-urban gradient in the city of Angers, France. Urban Forestry \& Urban Greening, 1(3), 19-27.

http://dx.doi.org/10.1016/j.ufug.2004.04.001.

Despois, J. (1953). Le Hodna. Paris: Éditions Presse Universitaires de France PUF.

Donadieu, P. (2005). Le paysage et les paysagistes, paysager n'est pas seulement jardiner. Paris: PUF.

Donégani, J. M., Michelat, G., \& Simon, M. (1980). Représentations du champ social, attitudes politiques et changements socio-économiques (Thèse de doctorat). Institut de Sociologie, Université de Lilles, Lille.

France. (1901). Loi 1901 relative à l'activité des associations: Naissance d'un organisme "service des cultures". Paris.

France. (1919). Loi Cornudet relative aux plans d'aménagements des villes. Paris.

France. (1943). Loi Prothin relative à une charte de l'urbanisme pour Paris. Paris.

France. (2014). Union nationale des entreprises du paysage. Paris: Unep.

Goodman, I. W. (1968). Principle and practice of urban planning. Washington: Institute for Training in Municipal Administration by the International City Mayors Association.

Hafiane, A. (1992). Des références conceptuelles de l'habitat informel, in croissance urbaine au Maghreb. Paris: Éditions PubliSud.

Hdjeidj, A, Chaline, C., \& Duboi-Maury, J. (2003). Alger, les nouveaux défis de l'urbanisation. Paris: L'Harmattan.

Ibn Hawkal, A. (1842). Description de l'Afrique (Traduction de Slane). Paris: Journal Asiatique.

Kettaf, F. (2015). La fabrique des espaces publics - Conceptions, formes et usages des places d'Oran Algérie. Les Cahiers d'Études sur le Monde Arabe et la Méditerranée, (26), 35-37. http://dx.doi.org/10.4000/emam.995.

Khoudour, M. (1999). Réalité des espaces verts urbains en Algérie. Cas d'étude ville de M’sila. In Actes du Séminaire International sur la Gestion des Villes (pp. 116-124). Msila: Presses de L'Université de M'sila.

Lamri, S. (2012). Espace vert urbain et périurbain de Sétif: État des lieux et place dans la gestion municipale (Mémoire de magistère). Département D’Architecture, Université Ferhat Abbas de Sétif, Algérie.

Laurie, M. (1986). An introduction to landscape architecture. New York: Elsevier Science Publishing.

Machon, N. (2011). Sauvage de ma rue: guide des plantes sauvages des villes de la région parisienne. Paris: Le Passage.

Malverti, X., \& Pinon, P. (1997). La ville régulière, modèle et tracés. Paris: Picard.

Merlin, P., \& Choay, F. (1996). Dictionnaire de l'urbanisme et de l'aménagement. Paris: PUF.

Mezrag, H. (2015). Le logement social collectif: entre la conception et l'usage. Cas de la ville de m'sila (Thèse de doctorat). Département D’Architecture, Université de Biskra, Algérie.

Mili, M. (2002). L'espace vert entre nécessité et enjeux cas des espaces verts de la ville de M'sila (Mémoire de magister). Institut de Gestion des Techniques Urbaines, Université de M’sila, Algérie.

Mili, M. (2018). Spécificités socio spatiales du paradigme du logement social transformé en copropriété. Cas de la ville de M'sila (Thèse de doctorat). Département D'Architecture, Université de Biskra, Algérie.

Mili, M., \& Boutabba, H. (2013). L'avenir des espaces verts dans l'environnement territorial, semi aride, du Hodna en Algérie. In: 7TH World Environmental Education Congress (pp. 450-465). Marrakech: Fondation Mohammed VI pour la Protection de L'Environnement.

Mili, M., Farhi, A., \& Boutabba, H. (2016). Évaluation post-occupationnelle des logements sociaux transformés en copropriété. Cas de la ville de M'sila en Algérie. Courrier du Savoir Scientifique et Technique, 20, 141-158.

Mimoune, S. (1999). Le cadre de vie dans la ville de M'sila. Diagnostic et mesures de protection. In: Actes du Séminaire International sur la Gestion des Villes (pp. 149-155). Msila: Presses de L'université de M’sila. 
Muret, J. P., Allain, Y. M., \& Sabrié, M. L. (1987). Les espaces urbains: concevoir, réaliser, gérer. Paris: Editions du Moniteur.

Oueslati, W., Madariaga, N., \& Salanié, J. (2008). Évaluation contingente d'aménités paysagères liées à un espace vert urbain. Une application au cas du parc Balzac de la ville d'Angers. Revue d'Etudes en Agriculture et Environnement, 87, 77-99.

Planel, A. (1980). La commune et l'aménagement des sites. Paris: Berger-Levrault.

Reygrobellet, B. (2007). La nature dans la ville: biodiversité et urbanisme (Étude du Conseil Economique et Social). Paris: Éditions des Journaux Officiels.

Rosso. (2018). INFO 568A M'sila. Récupéré le 1 janvier 2018, https://jeanyvesthorrignac.fr/wa_files/info_568a_msila.pdf.

Sebhi, S. (1987). Mutations du monde rural algérien le Hodna. Alger: Office des Publications Universitaires. Sétif. (2010). Plan directeur de l'aménagement et de l'urbanisme de la ville de M'sila (PDAU). Sétif: Agence URBA.

Zucchelli, A. (1984). Introduction à l'urbanisme opérationnel et la composition urbaine (3e. ed., vol. 2). Alger: Éditions OPU.

Editor: Paulo Nascimento Neto

Received: Jul. 21, 2018

Approved: Jan. 21, 2019 